PROCEEDINGS OF THE

AMERICAN MATHEMATICAL SOCIETY

Volume 126, Number 4, April 1998, Pages 1049-1054

S 0002-9939(98)04209-9

\title{
A BANACH COUPLE IS DETERMINED BY THE COLLECTION OF ITS INTERPOLATION SPACES
}

\author{
O. E. TIKHONOV AND L. V. VESELOVA
}

(Communicated by Palle E. T. Jorgensen)

\begin{abstract}
We prove that an arbitrary Banach couple is uniquely determined by the collection of all its interpolation spaces, which extends a result by $\mathrm{N}$. Aronszajn and E. Gagliardo.
\end{abstract}

\section{INTRODUCTION AND NOTATION}

In this paper, we affirmatively answer a question which originates from the classical paper by N. Aronszajn and E. Gagliardo [1]: is an arbitrary Banach couple uniquely determined by the collection of all its interpolation spaces? It was emphasized in the survey [3] (on page 9) that this question is an important unsolved problem from the fundamentals of the theory of interpolation of linear operators. In the monograph [2], Yu. A. Brudnyı̆ and N. Ya. Kruglyak conjectured that the answer to the question is affirmative (Conjecture 2.2.32 on page 133) and posed the corresponding problem (Problem 2.7.4(a) on page 286). Moreover, by using our techniques, we obtain a much-simplified proof of the uniqueness for embedded couples (cf. [1, p. 97, Theorem 10.XVI] and [2, p. 133, Theorem 2.2.33]).

We first introduce some notation and recall some definitions and facts from the theory of interpolation of linear operators in Banach spaces.

For a Banach space $E$, we will denote by $\|\cdot\|_{E}$ the norm in $E$, by $B_{E}$ the open unit ball in $E$, by $\bar{D}^{E}$ the closure in $E$ of a subset $D \subset E$, and by $\|\cdot\|^{E}$ the norm in the conjugate space $E^{*}$.

Let $E$ and $F$ be two Banach spaces. The notation $E \hookrightarrow F$ will mean that $E$ is linearly and continuously embedded into $F$. If $E \hookrightarrow F$ and $\varphi \in F^{*}$, then $\left.\varphi\right|_{E} \in E^{*}$, and we write $\|\varphi\|^{E}$ to denote $\left\|\left.\varphi\right|_{E}\right\|^{E}$. The notation $E \simeq F$ stands for the case when $E$ and $F$ coincide as linear spaces and their norms are equivalent.

Two Banach spaces $X$ and $Y$ form a Banach couple $(X, Y)$ if they both are linearly and continuously embedded into a certain Hausdorff topological vector space. Each Banach couple $(X, Y)$ can be canonically associated with two Banach spaces, the intersection $X \cap Y$ and the sum $X+Y$, with the norms defined as $\|z\|_{X \cap Y}=\max \left\{\|z\|_{X},\|z\|_{Y}\right\}(z \in X \cap Y)$ and $\|z\|_{X+Y}=\inf \left\{\|x\|_{X}+\|y\|_{Y}: x \in\right.$ $X, y \in Y, z=x+y\}(z \in X+Y)$. A Banach couple $(X, Y)$ is called embedded if $X \hookrightarrow Y$ or $Y \hookrightarrow X$. It is called regular if $X \cap Y$ is dense in $X$ and $Y$.

Received by the editors November 7, 1995 and, in revised form, September 23, 1996.

1991 Mathematics Subject Classification. Primary 46B70; Secondary 46M35.

Key words and phrases. Banach couple, interpolation space.

Supported by the Russian Foundation for Basic Research, grant no. 95-01-00025. 
A Banach space $Z$ is called intermediate for a Banach couple $(X, Y)$ if $X \cap Y \hookrightarrow$ $Z \hookrightarrow X+Y$.

A linear operator $T$ in $X+Y$ is called a linear continuous operator on a Banach couple $(X, Y)$ if $T$ maps $X$ into $X$ and $Y$ into $Y$ continuously. We denote by $L((X, Y))$ the set of all such operators. $L((X, Y))$ is a Banach space with respect to the norm

$$
\|T\|_{L((X, Y))}=\max \left\{\|T\|_{X \rightarrow X},\|T\|_{Y \rightarrow Y}\right\} .
$$

An intermediate space $Z$ for a Banach couple $(X, Y)$ is called an interpolation space for this Banach couple if $T(Z) \subset Z$ for all $T \in L((X, Y))$. The set of all interpolation spaces for $(X, Y)$ will be denoted by $\operatorname{Int}(X, Y)$. It is well-known $[1, \mathrm{p}$. 73, Theorem $6 . \mathrm{XI}]$ that if $Z \in \operatorname{Int}(X, Y)$, then there exists a constant $C$ such that the following "interpolation inequality" holds true:

$$
\|T\|_{Z \rightarrow Z} \leq C\|T\|_{L((X, Y))} \quad \text { for all } T \in L((X, Y)) .
$$

Let $(X, Y)$ and $(V, W)$ be two Banach couples. We will write $(X, Y) \sim(V, W)$ if $X \cap Y \simeq V \cap W$ and $X+Y \simeq V+W$. We will write $(X, Y) \simeq(V, W)$ if $X \simeq V$ and $Y \simeq W$ or $X \simeq W$ and $Y \simeq V$.

\section{LeMmaS}

We continue by presenting a sequence of lemmas, which we will use in the proof of the main result.

Lemma 2.1 (e.g., [4]). Let $(X, Y)$ be a Banach couple. Then

a) $X \cap Y$ is dense in $X$ if and only if $Y$ is dense in $X+Y[4$, p. 23, Corollary 1 of Lemma I.3.1];

b) if $X \cap Y$ is dense in both $X$ and $Y$, then it is dense in $X+Y$, too [4, p. 23, Corollary 2 of Lemma I.3.1].

Lemma 2.2 (e.g., [4, p. 25, Lemma I.3.4]). For a Banach couple $(X, Y)$, let a subset $D \subset X \cap Y$ be dense in $X$, and let $\|x\|_{Y} \leq c\|x\|_{X}$ on $D$. Then $X$ is embedded into $Y$ with an embedding constant less than or equal to $c$.

Let us remark that Lemma 2.2 is trivial to prove if $D$ is a linear space, and only this case is necessary in the present paper. The next lemma is immediate.

Lemma 2.3 (see [1, p. 96, the proof of Corollary 10.XIV]). Suppose that for two Banach couples $(X, Y)$ and $(V, W)$ the following conditions are fulfilled:

1) $(X, Y) \sim(V, W)$,

2) $X \hookrightarrow V$,

3) $Y \hookrightarrow W$.

Then $X \simeq V$ and $Y \simeq W$.

Lemma 2.4 ([1, p. 76, Theorem 7.IV]). If $Z \in \operatorname{Int}(X, Y)$, then $Z$ must satisfy one of the following four conditions:

1) $Z \simeq X+Y$,

2) $X \hookrightarrow Z \hookrightarrow \bar{X}^{X+Y}$,

3) $Y \hookrightarrow Z \hookrightarrow \bar{Y}^{X+Y}$,

4) $X \cap Y \hookrightarrow Z \hookrightarrow \frac{,}{X \cap Y} X+Y$.

Lemma 2.5 will play a leading role in the proof of the main result. 
Lemma 2.5. Let $E$ and $F$ be Banach spaces such that $E \hookrightarrow F$. Suppose that $E$ and $F$ do not coincide as linear spaces. Then there exists a sequence $\left\{\varphi_{n}\right\} \subset F^{*}$ such that $\left\|\varphi_{n}\right\|^{F}=1$ and $\left\|\varphi_{n}\right\|^{E} \rightarrow 0$.

Proof. It is well-known (e.g., [4, p. 11, Lemma I.1.1]) that the unit ball $B_{E}$ is nowhere dense in $F$. Therefore, ${\overline{n B_{E}}}^{F}$ does not contain $B_{F}$ for any natural number $n$. Hence, there exists $x_{n} \in B_{F}$ such that $x_{n} \notin{\overline{n B_{E}}}^{F}$. It follows from the HahnBanach theorem that there exists $\varphi_{n} \in F^{*}$ such that $\left\|\varphi_{n}\right\|^{F}=1$ and

$$
\sup \left\{\left|\varphi_{n}(x)\right|: x \in{\overline{n B_{E}}}^{F}\right\}<\varphi_{n}\left(x_{n}\right) .
$$

Hence we have

$$
\left\|\varphi_{n}\right\|^{E}=\sup \left\{\left|\varphi_{n}(x)\right|: x \in B_{E}\right\} \leq \varphi_{n}\left(x_{n}\right) / n \leq 1 / n .
$$

\section{MAIN RESUlts}

Note, first, that the following implications hold true for every pair of Banach couples $(X, Y)$ and $(V, W)$ :

$$
\begin{aligned}
\operatorname{Int}(X, Y)=\operatorname{Int}(V, W) & \Longrightarrow(X, Y) \sim(V, W), \\
\operatorname{Int}(V, W) \subset \operatorname{Int}(X, Y) & \Longleftrightarrow V, W \in \operatorname{Int}(X, Y) .
\end{aligned}
$$

The proof is straightforward.

Next, we recall a result which is due to N. Aronszajn and E. Gagliardo (see [1, p. 97 , Theorem 10.XVI]. Here, we give a proof which is simpler than in [1] or [2, p. 133, Theorem 2.2.33].

Theorem 3.1 ([1]). Let a Banach couple $(X, Y)$ be non-embedded. Then either $X$ or $Y$ does not belong to $\operatorname{Int}(X \cap Y, X+Y)$.

Proof. We denote $\Delta=X \cap Y$ and $\Sigma=X+Y$. Suppose that $(X, Y)$ is nonregular and $X, Y \in \operatorname{Int}(\Delta, \Sigma)$. Then, from Lemma 2.4, we get $\Delta \hookrightarrow X \hookrightarrow \bar{\Delta}^{\Sigma}$ and $\Delta \hookrightarrow Y \hookrightarrow \bar{\Delta}^{\Sigma}$. Hence $\Sigma \hookrightarrow \bar{\Delta}^{\Sigma}$. By Lemma 2.1 a), this contradicts the assumption that $(X, Y)$ is not regular.

Now, let $(X, Y)$ be regular. Note that $\bar{\Delta}^{\Sigma}=\Sigma$ by Lemma $\left.2.1 \mathrm{~b}\right)$. Suppose, for example, that $Y \in \operatorname{Int}(\Delta, \Sigma)$. By Lemma 2.5 , we can choose a sequence $\left\{\varphi_{n}\right\} \subset \Sigma^{*}$ with $\left\|\varphi_{n}\right\|^{\Sigma}=1$ and $\left\|\varphi_{n}\right\|^{X} \rightarrow 0$. Clearly, $\left\|\varphi_{n}\right\|^{\Delta} \rightarrow 0$. Since $B_{\Sigma}=\operatorname{conv}\left(B_{X} \cup B_{Y}\right)$, we have $\left\|\varphi_{n}\right\|^{Y} \rightarrow 1$. For $x \in \Delta$, we consider the linear operators of rank one given by the formula $T_{n}(\cdot)=\varphi_{n}(\cdot) x$. Then we have

$$
\begin{gathered}
\left\|T_{n}\right\|_{Y \rightarrow Y}=\left\|\varphi_{n}\right\|^{Y}\|x\|_{Y} \rightarrow\|x\|_{Y}, \\
\left\|T_{n}\right\|_{\Delta \rightarrow \Delta}=\left\|\varphi_{n}\right\|^{\Delta}\|x\|_{\Delta} \rightarrow 0, \\
\left\|T_{n}\right\|_{\Sigma \rightarrow \Sigma}=\left\|\varphi_{n}\right\|^{\Sigma}\|x\|_{\Sigma}=\|x\|_{\Sigma} .
\end{gathered}
$$

By applying the interpolation inequality for $Y \in \operatorname{Int}(\Delta, \Sigma)$, we infer that there exists a constant $c$ such that $\|x\|_{Y} \leq c\|x\|_{\Sigma}$ for all $x \in \Delta$. By Lemma 2.2, it follows that $\Sigma \hookrightarrow Y$. This contradicts the hypothesis of the theorem of that $(X, Y)$ is not embedded.

Corollary 3.1. Suppose that for an embedded Banach couple $(X, Y)$ and another Banach couple $(V, W)$ we have $\operatorname{Int}(X, Y)=\operatorname{Int}(V, W)$. Then $(X, Y) \simeq(V, W)$. 
Theorem 3.2. Suppose that for a Banach couple $(X, Y)$ and a non-embedded Banach couple $(V, W)$ we have $(X, Y) \sim(V, W)$ and $\operatorname{Int}(V, W) \subset \operatorname{Int}(X, Y)$. Then $(X, Y) \simeq(V, W)$.

Proof. We first remark that the hypotheses of this theorem imply, using Theorem 3.1 , that $(X, Y)$ cannot be an embedded couple. Now the proof naturally splits into three parts.

I. Let us first consider the case when $X \cap Y$ is dense in neither $X$ nor $Y$. By Lemma $2.1 \mathrm{a})$, neither $X$ nor $Y$ is dense in $X+Y$. Since they are both $\operatorname{in} \operatorname{Int}(X, Y)$, each of the spaces $V$ and $W$ must satisfy at least one of the conditions 2), 3) or 4) of Lemma 2.4. If they are both contained in $\bar{X}^{X+Y}$, then so is $V+W$, which contradicts the assumptions that $V+W \simeq X+Y$ and $\bar{X}^{X+Y} \nsucceq X+Y$. Similarly, they cannot both be contained in $\bar{Y}^{X+Y}$. Thus neither $V$ nor $W$ can satisfy condition 4), and the only remaining possibilities are that either

$$
X \hookrightarrow V \hookrightarrow \bar{X}^{X+Y} \text { and } Y \hookrightarrow W \hookrightarrow \bar{Y}^{X+Y}
$$

or a similar condition holds with $V$ and $W$ interchanged. In both of these cases it follows from Lemma 2.3 that $(X, Y) \simeq(V, W)$.

II. We now suppose that $X \cap Y$ is dense in $Y$, but not in $X$. Then $X$ is dense in $X+Y$, but $Y$ is not. Again $V$ and $W$ must each satisfy at least one of conditions 2), 3) or 4) of Lemma 2.4. Exactly as in the previous step, they cannot both be contained in $\bar{Y}^{X+Y}$. Also they cannot both satisfy 2), since $X$ cannot be contained in $V \cap W \simeq X \cap Y$. Thus exactly one of them must satisfy 2$)$. If the other satisfies $3)$, then again Lemma 2.3 ensures that $(X, Y) \simeq(V, W)$. It follows that we can assume that

$$
X \cap Y \hookrightarrow V \hookrightarrow \overline{X \cap Y}^{X+Y}, \quad X \hookrightarrow W \hookrightarrow \bar{X}^{X+Y}
$$

(or that an analogous condition holds with the roles of $V$ and $W$ interchanged).

We will consider two different cases, a) and b).

a) Let $X+V \simeq X+Y$. By Lemma 2.5, we can choose a sequence $\left\{\varphi_{n}\right\} \subset(X+Y)^{*}$ such that $\left\|\varphi_{n}\right\|^{X+Y}=1$ and $\left\|\varphi_{n}\right\|^{X} \rightarrow 0$. Then $\left\|\varphi_{n}\right\|^{Y} \rightarrow 1$. Since $X+V \simeq X+Y$ and $\left\|\varphi_{n}\right\|^{X} \rightarrow 0$, there exist constants $c_{1}, c_{2}>0$ and a natural number $n_{0}$ such that $c_{1} \leq\left\|\varphi_{n}\right\|^{V} \leq c_{2}\left(n \geq n_{0}\right)$. For $x \in X \cap Y$ and the operators $T_{n}(\cdot)=\varphi_{n}(\cdot) x$, we have

$$
\begin{gathered}
\left\|T_{n}\right\|_{X \rightarrow X}=\left\|\varphi_{n}\right\|^{X}\|x\|_{X} \rightarrow 0, \\
\left\|T_{n}\right\|_{Y \rightarrow Y}=\left\|\varphi_{n}\right\|^{Y}\|x\|_{Y} \rightarrow\|x\|_{Y}, \\
\left\|T_{n}\right\|_{V \rightarrow V}=\left\|\varphi_{n}\right\|^{V}\|x\|_{V} \geq c_{1}\|x\|_{V} \quad\left(n \geq n_{0}\right) .
\end{gathered}
$$

By applying the interpolation inequality for $V \in \operatorname{Int}(X, Y)$, we obtain $\|x\|_{V} \leq$ $c\|x\|_{Y}$ for all $x \in X \cap Y$. Hence by Lemma 2.2, we have $Y \hookrightarrow V$. As $X \hookrightarrow W$, we obtain from Lemma 2.3 that $X \simeq W$ and $Y \simeq V$.

b) We now consider the case when $X+V$ does not coincide with $X+Y$ as a linear space. We choose a sequence $\left\{\varphi_{n}\right\} \subset(X+Y)^{*}$ such that $\left\|\varphi_{n}\right\|^{X+Y}=1$ and $\left\|\varphi_{n}\right\|^{X+V} \rightarrow 0$. Clearly, $\left\|\varphi_{n}\right\|^{X} \rightarrow 0$ and $\left\|\varphi_{n}\right\|^{V} \rightarrow 0$. Hence, $\left\|\varphi_{n}\right\|^{Y} \rightarrow 1$, and there exists a constant $c_{1}>0$ such that $\left\|\varphi_{n}\right\|^{W} \geq c_{1}$ if $n$ is sufficiently large. For $x \in X \cap Y$, consider the operators $T_{n}(\cdot)=\varphi_{n}(\cdot) x$. We have

$$
\left\|T_{n}\right\|_{X \rightarrow X}=\left\|\varphi_{n}\right\|^{X}\|x\|_{X} \rightarrow 0
$$




$$
\begin{gathered}
\left\|T_{n}\right\|_{Y \rightarrow Y}=\left\|\varphi_{n}\right\|^{Y}\|x\|_{Y} \rightarrow\|x\|_{Y}, \\
\left\|T_{n}\right\|_{W \rightarrow W}=\left\|\varphi_{n}\right\|^{W}\|x\|_{W} .
\end{gathered}
$$

By applying the interpolation inequality for $W$, we obtain $\|x\|_{W} \leq c\|x\|_{Y}$ for all $x \in X \cap Y$. From this, we conclude that $Y \hookrightarrow W$. As $X \hookrightarrow W$, this implies that $X+Y \hookrightarrow W$, which contradicts the hypothesis of the theorem that $(V, W)$ is not embedded.

III. Let $(X, Y)$ be regular. We first suppose that $X+V \simeq X+Y$. As in the case II a), we obtain $Y \hookrightarrow V$. If, in addition, $Y+W \simeq X+Y$, then we see (similarly to the case II a)) that $X \hookrightarrow W$. By Lemma 2.3, we obtain $X \simeq W$ and $Y \simeq V$. If $Y+W \not X+Y$, then, similarly to the case II b), we obtain $X \hookrightarrow V$. Since $Y \hookrightarrow V$ and $X+Y \simeq V+W$, this contradicts the hypothesis that the couple $(V, W)$ is not embedded.

Now, we suppose that $X+V \nsucceq X+Y$. As in the case II b), we obtain that $Y \hookrightarrow W$. Similarly to the case II a), from $Y+W \simeq X+Y$ we get $X \hookrightarrow W$, which contradicts the hypothesis that the couple $(V, W)$ is not embedded. Therefore, $Y+W \not X+Y$. Then $X \hookrightarrow V$, and by Lemma 2.3, we have $X \simeq V$ and $Y \simeq W$.

Corollary 3.2. Let $(X, Y)$ and $(V, W)$ be two Banach couples such that $\operatorname{Int}(X, Y)$ $=\operatorname{Int}(V, W)$. Then $(X, Y) \simeq(V, W)$.

Remark 3.1. Theorem 3.2 gives an answer to a question which was treated by N. Aronszajn and E. Gagliardo in [1]. In that work, they stated (see Remark 10.XV on page 96) that they did not know whether there exist two different non-embedded Banach couples, $(X, Y)$ and $(V, W)$, such that $(X, Y) \sim(V, W)$ and $\operatorname{Int}(V, W) \subset$ $\operatorname{Int}(X, Y)$.

Remark 3.2. By making use of Corollary 3.2, we have proved in [5],[6] that a Banach couple is uniquely determined by the collection of all its exact interpolation spaces. The first step in that direction was made in [7]. In that paper, the case of a Banach couple $(X, Y)$ with $X \simeq Y$ was examined. Note that in case of the collection of the exact interpolation spaces the meaning of "uniquely determined" differs from that in the present paper.

\section{ACKNOWLEDGMENT}

We are grateful to N. M. Zobin, who attracted our attention to inverse interpolation problems, and to Yu. A. Brudnyı̆, whose interest has stimulated our work.

We are highly indebted to our referee for making many valuable corrections and suggestions.

\section{REFERENCES}

1. N. Aronszajn and E. Gagliardo, Interpolation spaces and interpolation methods, Ann. Mat. Pure ed Appl. 68 (1965), 51-117. MR 37:1951

2. Yu. A. Brudnyı̆ and N. Ya. Kruglyak, Interpolation functors and interpolation spaces I, NorthHolland, 1991. MR 93b:46141

3. Yu. A. Brudnyı̆, S. G. Kreĭn, and E. M. Semenov, Interpolation of linear operators, in "Itogi Nauki i Tekhniki. Matem. Analiz" 24, Moscow, 1986, pp. 3-163 (Russian); English transl., J. of Soviet Math. 42 (1988), 2009-2113. MR 88e:46056

4. S. G. Kreı̆n, Yu. I. Petunin, and E. M. Semenov, Interpolation of linear operators, "Nauka", Moscow, 1978 (Russian); English transl., Amer. Math. Soc., Providence, 1982. MR 84j:46103 
5. L. V. Veselova and O. E. Tikhonov, The uniqueness of the solution to inverse interpolation problems, Research Institute of Mathematics and Mechanics, Preprint no. 95-2, Kazan Mathematics Foundation, Kazan, 1995 (Russian).

6. O. E. Tikhonov and L. V. Veselova, The uniqueness of the solution to the inverse problem of exact interpolation (submitted to the Proceedings of the International Workshop on Function Spaces, Interpolation Spaces and Related Topics).

7. L. V. Veselova and O. E. Tikhonov, On the uniqueness of the solution of the inverse exact interpolation problem, Funktsional'nyi Analiz i ego Prilozheniya 26 (2) (1992), 67-68 (Russian); English transl., Funct. Anal. Appl. 26 (2) (1992), 129-131. MR 93g:46076

Research Institute of Mathematics and Mechanics, Kazan State University, UniverSitetskaya Str. 17, Kazan, Tatarstan, 420008 Russia

E-mail address: Oleg.Tikhonov@ksu.ru

Department of Higher Mathematics, Kazan State Technological University, Karl Marx Str. 68, Kazan, Tatarstan, 420015 Russia 